\title{
NEW SYSTEMS OF HYPERGEODESICS DEFINED ON A SURFACE
}

\author{
P. O. BELL
}

Introduction. Let a non-ruled surface $S$ be referred to its asymptotic net as parametric. As a point $P_{y}$ moves along a curve $C_{\lambda}$ of $S$, the tangents at $P_{y}$ to the $u$-and $v$-asymptotic curves of $S$ describe two ruled surfaces $R_{\lambda}^{u}$ and $R_{\lambda}^{v}$, respectively. Let $S_{\rho}$ and $S_{\sigma}$ denote arbitrary transversal surfaces of the congruences of $u$ - and $v$-tangents of $S$, respectively. The purpose of the present paper is to introduce and study systems of curves of $S$ which will be called $\rho$ - and $\sigma$-tangeodesics.

Definition. $A$ curve $C_{\lambda}$ of $S$ whose associated ruled surface $R_{\lambda}^{u}$ intersects the surface $S_{\rho}$ in an asymptotic curve of $R_{\lambda}^{u}$ is a $\rho$-tangeodesic of $S$. Similarly, a curve $C_{\lambda}$ of $S$ whose associated ruled surface $R_{\lambda}^{0}$ intersects $S_{\sigma}$ in an asymptotic curve of $R_{\lambda}^{0}$ is a $\sigma$-tangeodesic of $S$.

The $\rho$ - and $\sigma$-tangeodesics of $S$ at $P_{y}$ are found to be associated in remarkable manners with the edges of Green, the directrices of Wilczynski, and the projective normal of Fubini. In fact, a new geometric characterization is obtained for each of these lines.

1. Tangeodesics. If the parametric net on a non-ruled surface $S$ is the asymptotic net, the homogeneous projective coordinates $y^{(i)}(u, v)$ $(i=1,2,3,4)$ of a general point $P_{y}$ of $S$ are solutions of a system of differential equations which may be assumed to be reduced to Wilczynski's canonical form

$$
y_{u u}+2 b y_{v}+f y=0, \quad y_{v v}+2 a^{\prime} y_{u}+g y=0 .
$$

The homogeneous coordinates of points $\rho, \sigma$ on arbitrarily selected transversal surfaces $S_{\rho}$ and $S_{\sigma}$ of the congruences of $u$ - and $v$-tangents of $S$ are given by the vector forms

$$
\rho=y_{u}-\beta y, \quad \sigma=y_{v}-\alpha y,
$$

wherein $\beta, \alpha$ are arbitrary analytic functions of $u, v$.

Let $l$ denote the line joining $\rho, \sigma$ and let $l^{\prime}$ denote its reciprocal at $P_{y}$. The line $l^{\prime}$ joins the points $P_{y}$ and $z$ where $z$ is given by

$$
z=y_{u v}-\alpha y_{u}-\beta y_{v}
$$

in which $\beta$ and $\alpha$ are the functions in (1.2). The line $l$, according to Green's classification, is a line of the first kind and generates a con-

Received by the editors February 20, 1943. 
gruence $\Gamma$ of the first kind as $P_{y}$ moves over $S$. The line $l^{\prime}$ is a line of the second kind and generates a congruence $\Gamma^{\prime}$ of the second kind as $P_{y}$ moves over $S$.

Let $C_{\lambda}$ denote an integral curve of the curvilinear differential equation

$$
d v-\lambda(u, v) d u=0 .
$$

Regarding $u$ as independent variable we write $v^{\prime}=\lambda(u, v)$ and $v^{\prime \prime}=\lambda_{u}+\lambda \lambda_{v}$, in which accents indicate differentiation with respect to $u$.

The homogeneous coordinates of a general point of the ruled surface $R_{\lambda}^{u}$ are represented by the vector form

$$
\bar{y}=y_{u}+w y,
$$

wherein $u$ and $w$ are independent variables and $v$ varies in accordance with the relation $v^{\prime}=\lambda(u, v)$.

Let us put $u=u(t), w=w(t)$, so that $\bar{y}$ describes a curve on $R_{\lambda}^{u}$ as $t$ varies. The necessary and sufficient condition that this curve be an asymptotic curve of $R_{\lambda}^{u}$ is that the determinant equation

$$
\left(\bar{y}, \bar{y}_{u}+v^{\prime} \bar{y}_{v}, \bar{y}_{w}, d^{2} \bar{y} / d t^{2}\right)=0
$$

be satisfied. If we transform equation (1.6) by making use of equations (1.5), (1.4) and (1.1) we obtain, in view of the inequality $\left(y, y_{u}, y_{v}, y_{u v}\right) \neq 0$, the equation

$$
d w / d u=\left\{2 b^{2}+\left(b_{u}-2 b w\right) v^{\prime}+\left(w^{2}+2 b_{v}+f\right) v^{\prime 2}-2 a^{\prime} b v^{\prime 3}-b v^{\prime \prime}\right\} / v^{\prime 2} .
$$

As $P_{y}$ moves along $C_{\lambda}$ the point $\rho$ moves in the direction defined by (1.7) if and only if $w=-\beta$ satisfies (1.7). To obtain, therefore, the curvilinear differential equation for the $\rho$-tangeodesics we have merely to substitute $-\beta$ for $w$ in (1.7) and clear of fractions. The result is

$$
b v^{\prime \prime}-2 b^{2}-\left(2 b \beta+b_{u}\right) v^{\prime}-\left(\beta^{2}+2 b_{v}+f+\beta_{u}\right) v^{\prime 2}+\left(2 a^{\prime} b-\beta_{v}\right) v^{\prime 3}=0 .
$$

The differential equation for the $\sigma$-tangeodesics may be obtained by making the substitution

$$
\left(\begin{array}{ccccccc}
v^{\prime \prime} & v^{\prime} & v & b & a^{\prime} & \beta & f \\
-v^{\prime \prime} / v^{\prime 3} & 1 / v^{\prime} & u & a^{\prime} & b & \alpha & g
\end{array}\right)
$$

in (1.8). The result, on simplifying, is

(1.9) $a^{\prime} v^{\prime \prime}+\alpha_{u}-2 a^{\prime} b+\left(\alpha^{2}+2 a_{u}^{\prime}+g+\alpha_{v}\right) v^{\prime}+\left(2 a^{\prime} \alpha+a_{v}^{\prime}\right) v^{\prime 2}+2 a^{\prime 2} v^{\prime 3}=0$.

2. Systems of hypergeodesics which have no cusp-axes. The curves defined on a surface $S$ by a differential equation of the form 


$$
v^{\prime \prime}=A+B v^{\prime}+C v^{\prime 2}+D v^{\prime 3}
$$

in which the coefficients are functions of $u, v$ and accents indicate differentiation with respect to the independent variable $u$, are called hypergeodesics. ${ }^{1}$ The envelope of the osculating planes at a point $P_{y}$ of the hypergeodesics (2.1) is a cone which is ordinarily of the third class. When this cone is of the third class it has three distinct cusp-planes which intersect in a line called the cusp-axis of the cone, or the cuspaxis of the hypergeodesics at the point $P_{y}$. The cusp-axis is the line $l^{\prime}$ for which $\alpha$ and $\beta$ are given by

$$
\alpha=C / 2, \quad \beta=-B / 2 .
$$

We are interested here in those cases in which the class of the cone is less than three and the cone has no cusp-axis. The local equation of the osculating plane at $P_{y}$ of the curve $C_{\lambda}$ defined by (1.4) is

$$
2 \lambda\left(\lambda x_{2}-x_{3}\right)+\left(\lambda^{\prime}-2 b+2 a^{\prime} \lambda^{3}\right) x_{4}=0,
$$

when referred to the tetrahedron whose vertices have the general coordinates $y, y_{u}, y_{v}, y_{u v}$. Assuming $C_{\lambda}$ to be an integral curve of (2.1) we replace $\lambda^{\prime}$ by the right member of (2.1) and put $\lambda$ in place of $v^{\prime}$. The result is

$$
2 \lambda\left(\lambda x_{2}-x_{3}\right)+\left(A-2 b+B \lambda+C \lambda^{2}+\left[D+2 a^{\prime}\right] \lambda^{3}\right) x_{4}=0 .
$$

The union curves of a congruence $\Gamma^{\prime}$ form a system of hypergeodesics, sometimes called an axial system, whose osculating planes at $P_{y}$ all pass through the line $l^{\prime}$ of the congruence $\Gamma^{\prime}$. Equation (2.1) represents such a system if $A=2 b$ and $D=-2 a^{\prime}$.

A system of hypergeodesics (2.1) for which

$$
A=2 b, \quad D \neq-2 a^{\prime}
$$

will be called, for reasons which appear later, a u-polar system; and a system (2.1) for which

$$
D=-2 a^{\prime}, \quad A \neq 2 b
$$

will be called a $v$-polar system.

If system (2.1) is a $u$-polar system, the equation for the envelope of its osculating planes at $P_{y}$ may be readily found from (2.4) to be

$$
\left(2 x_{2}+C x_{4}\right)^{2}-4\left(D+2 a^{\prime}\right)\left(B x_{4}-2 x_{3}\right) x_{4}=0 .
$$

Similarly, if (2.1) represents a $v$-polar system the equation for the envelope of its osculating planes at $P_{y}$ may be found to be

${ }^{1} \mathrm{G}$. Fubini, Fondamenti della geometria proiettivo-differenziale di una superficie, Atti Accad. Sci. Torino vol. 53 (1918) p. 1034. 


$$
\left(2 x_{3}-B x_{4}\right)^{2}-4(A-2 b)\left(2 x_{2}+C x_{4}\right) x_{4}=0 .
$$

Since the cones (2.7) and (2.8) are nondegenerate quadric cones, they have no cusp-axes at $P_{y}$. Hence we have that neither a $u$-polar system nor a v-polar system of hypergeodesics has a cusp-axis at $P_{y}$.

There are two generators of the cone (2.7) which are such that the tangent planes of the cone along these generators pass through the $u$-tangent to $S$ at $P_{y}$. One of these is the $v$-tangent to $S$ at $P_{y}$ and the other is the line $l^{\prime}$ for which

$$
\alpha=C / 2, \quad \beta=-B / 2,
$$

wherein $B$ and $C$ are the functions appearing in (2.7). This line $l^{\prime}$ will be called the $u$-edge of the $u$-polar system.

The v-edge of a v-polar system is characterized similarly.

Since equations (2.9) are of the same form as equations (2.2), we have immediately this theorem.

THEOREM 2.1. If the coefficients $B$ and $C$ of the differential equation of a non-polar system of hypergeodesics are identical with the corresponding coefficients of the differential equation of a u-polar system of hypergeodesics, the cusp-axis of the non-polar system at $P_{y}$ coincides with the $u$-edge of the u-polar system at $P_{y}$.

A similar theorem applies, of course, to a v-polar system of hypergeodesics.

The forms of the differential equations (1.8) and (1.9) show clearly that the $\rho$ - and $\sigma$-tangeodesics form $u$ - and $v$-polar systems of hypergeodesics. For the system (1.8) we have

$$
\begin{gathered}
A=2 b, \quad B=2 \beta+b_{u} / b, \quad C=\left(\beta^{2}+2 b_{v}+f+\beta_{u}\right) / b, \\
D=\left(\beta_{v}-2 a^{\prime} b\right) / b .
\end{gathered}
$$

For the system (1.9) we have

$$
\begin{gathered}
A=\left(2 a^{\prime} b-\alpha_{u}\right) / a^{\prime}, \quad B=-\left(\alpha^{2}+2 a_{u}^{\prime}+g+\alpha_{v}\right) / a^{\prime}, \\
C=-2 \alpha-a_{v}^{\prime} / a^{\prime}, \quad D=-2 a^{\prime} .
\end{gathered}
$$

The cone (2.7) is associated with the system (1.8) of $\rho$-tangeodesics if $A, B, C, D$ are given by (2.10). Similarly, if $A, B, C, D$ are defined by (2.11), the cone (2.8) is associated with the system (1.9) of $\sigma$-tangeodesics.

The $u$-edge of the $\rho$-tangeodesics (1.8) is the line $l_{1}^{\prime}$ passing through the points $P_{y}$ and $z_{1}$, where $z_{1}$ is given by $z_{1}=y_{u v}-\alpha_{1} y_{u}-\beta_{1} y_{v}$, in which

$$
\beta_{1}=-\beta-b_{u} / 2 b, \quad \alpha_{1}=\left(\beta^{2}+2 b_{v}+f+\beta_{u}\right) / 2 b .
$$


The $v$-edge of the $\sigma$-tangeodesics is the line $l_{2}^{\prime}$ passing through $P_{y}$ and $z_{2}$ where $z_{2}$ is given by $z_{2}=y_{u v}-\alpha_{2} y_{u}-\beta_{2} y_{v}$, in which

$$
\beta_{2}=\left(\alpha^{2}+2 a_{u}^{\prime}+g+\alpha_{v}\right) / 2 a^{\prime}, \quad \alpha_{2}=-\alpha-a_{v}^{\prime} / 2 a^{\prime} .
$$

3. The edges of Green, the directrices of Wilczynski, and the projective normal. Let us apply the results of $\S \S 1$ and 2 to obtain new characterizations of the edges of Green, the directrices of Wilczynski, and the projective normal of Fubini. The plane which is tangent to the cone (2.7) of the $\rho$-tangeodesics along the $u$-edge intersects the plane which is tangent to the cone (2.8) of the $\sigma$-tangeodesics along the $v$-edge in a line $\bar{l}^{\prime}$ of the second kind which will be called the joint-edge of the systems of $\rho$ - and $\sigma$-tangeodesics of $S$ at $P_{y}$. This line passes through the points $P_{y}$ and $\bar{z}$ where the general coordinates of $\bar{z}$ are given by $\bar{z}=y_{u v}-\bar{\alpha} y_{u}-\bar{\beta} y_{v}$, in which

$$
\bar{\alpha}=-\alpha-a_{v}^{\prime} / 2 a^{\prime}, \quad \bar{\beta}=-\beta-b_{u} / 2 b .
$$

Since the functions $\alpha, \beta$ associated with the edges of Green are given by

$$
\alpha=-a_{v}^{\prime} / 4 a^{\prime}, \quad \beta=-b_{u} / 4 b,
$$

we have the following theorem.

THEOREM 3.1. The second edge of Green at $P_{y}$ lies in the plane $\pi$ determined by the joint-edge of the systems of $\rho$-and $\sigma$-tangeodesics of $S$ at $P_{y}$ and the reciprocal $l^{\prime}$ of the line $l$ joining $\rho, \sigma$. The joint-edge coincides with the line $l^{\prime}$ if and only if $l^{\prime}$ is the second edge of Green. Any two particular planes $\pi_{1}$ and $\pi_{2}$ of the plane $\pi$ (corresponding to selections $\rho_{1}, \sigma_{1}$ and $\left.\rho_{2}, \sigma_{2}\right)$ intersect in the second edge of Green.

Let $\sigma_{a}$ denote the intersection of the tangent plane to $S_{\rho}$ at $\rho$ with the $v$-tangent to $S$ at $P_{y}$ and let $\rho_{a}$ denote the intersection of the tangent plane to $S_{\sigma}$ at $\sigma$ with the $u$-tangent to $S$ at $P_{y}$. It may be easily verified that the general coordinates of $\rho_{a}$ and $\sigma_{a}$ are given by $\rho_{a}=y_{u}-\beta_{a} y, \sigma_{a}=y_{v}-\alpha_{a} y$, wherein $\beta_{a}, \alpha_{a}$ are given by

$$
\beta_{a}=-\left(g+\alpha_{v}+\alpha^{2}\right) / 2 a^{\prime}, \quad \alpha_{a}=-\left(f+\beta_{u}+\beta^{2}\right) / 2 b .
$$

The line $l_{a}$ joining $\rho_{a}, \sigma_{a}$ was introduced by the author in a previous paper $^{2}$ and called the asymptotic associate of the line $l$ joining $\rho, \sigma$.

The plane determined by the $v$-tangent of $S$ at $P_{y}$ and the $u$-edge of the $\rho$-tangeodesics at $P_{y}$ is the polar plane of the $u$-tangent of $S$ at $P_{y}$ with respect to the cone (2.7) of the $\rho$-tangeodesics. Similarly, the

${ }^{2}$ P. O. Bell, A study of curved surfaces by means of certain associated ruled surfaces, Trans. Amer. Math. Soc. vol. 46 (1939) p. 396. 
plane determined by the $u$-tangent of $S$ at $P_{y}$ and the $v$-edge of the $\sigma$-tangeodesics at $P_{y}$ is the polar plane of the $v$-tangent of $S$ at $P_{y}$ with respect to the cone (2.8) of the $\sigma$-tangeodesics. These two polar planes intersect in a line $l_{3}^{\prime}$ which will be called the polar-axis of the $\rho$ - and $\sigma$-tangeodesics at $P_{y}$. This line may be shown to pass through the points $P_{y}$ and $z_{3}$ where the general coordinates of $z_{3}$ are given by $z_{3}=y_{u v}-\alpha_{3} y_{u}-\beta_{3} y_{v}$, in which

$$
\alpha_{3}=-\alpha_{a}+b_{v} / b, \quad \beta_{3}=-\beta_{a}+a_{u}^{\prime} / a^{\prime} .
$$

Since the functions $\alpha, \beta$ for the directrix $l^{\prime}$ of Wilczynski are given by

$$
\alpha=b_{v} / 2 b, \quad \beta=a_{u}^{\prime} / 2 a^{\prime},
$$

equations (3.4) are such that we have, immediately, this theorem.

ThEOREM 3.2. The second directrix of Wilczynski lies in the plane $p$ determined by the polar-axis of the $\rho$-and $\sigma$-tangeodesics at $P_{y}$ and the reciprocal $l_{a}^{\prime}$, with respect to $S$ at $P_{y}$, of the asymptotic associate of $l$. Any two particular planes $p_{1}$ and $p_{2}$ of the plane $p$ (corresponding to lines $l_{1}$ and $\left.l_{2}\right)$ intersect in the second directrix of Wilczynski.

Theorems 3.1 and 3.2 may be dualized by replacing the lines and planes of these theorems by their reciprocals with respect to $S$ at $P_{y}$. The dual of Theorem 3.1 is the following theorem.

THEOREM 3.3. The first edge of Green contains the point $P$ of intersection of the reciprocal of the joint-edge of the systems of $\rho$ - and $\sigma$-tangeodesics of $S$ and the line $l$ joining $\rho, \sigma$. These three lines coincide if and only if the line $l$ is the first edge of Green. Any two particular points $P_{1}$ and $P_{2}$ of the point $P$ (corresponding to selections $\rho_{1}, \sigma_{1}$ and $\rho_{2}, \sigma_{2}$ ) determine the first edge of Green.

The statement of the dual of Theorem 3.2 will be left to the care of the reader.

Finally, since the projective-normal of $S$ at $P_{y}$ is the line for which the functions $\alpha, \beta$ are given by $\alpha=-\left(b_{v} / 2 b+a_{v}^{\prime} / 2 a^{\prime}\right), \beta=-\left(a_{u}^{\prime} / 2 a^{\prime}+b_{u} / 2 b\right)$, and the first directrix of $S$ at $P_{y}$ is the line $l$ for which $\alpha=b_{v} / 2 b$, $\beta=a_{u}^{\prime} / 2 a^{\prime}$, we have from equations (3.1) this theorem.

ThEOREM 3.4. If the line $l$ joining $\rho, \sigma$ is the first directrix of Wilczynski, the joint-edge of the systems of $\rho$ and $\sigma$-tangeodesics of $S$ at $P_{y}$ is the projective normal of Fubini.

UNIVERSITY OF KANSAS 